\title{
Development of Human Comfort Degree Forecast System
}

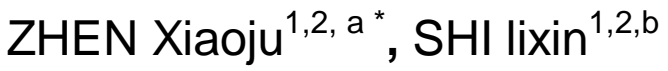 \\ ${ }^{1}$ Hebei Institute of Meteorology, Shijiazhuang,050021,China. \\ ${ }^{2}$ Key Laboratory of Meteorology and Ecological Environment of Hebei Province, Shijiazhuang, \\ 050021 China \\ axjzzhen@163.com, bshilixin@sina.com,
}

Keywords: Human comfort degree, forecast system, development.

Abstract. Based on interpreting Numerical Weather Prediction (NWP) products, using inverse distance weighting (IDW) method, the detailed human comfort degree forecast system is developed. The preliminary application shows better result.

\section{Introduction}

Human comfort is an indicator to judge whether climate condition is comfortable, which is based on the principle of heat balance between human body and its surrounding environment [1]. In the evaluation of living environment and tourism resources, human comfort degree has gradually become one of the most important factors. Study on the climate comfort can be traced back to the beginning of the nineteenth century [2,3]. Scientists in different periods were proposed wind chill index [4], the discomfort index [5], comfort index [6,7], temperature humidity index and wind effect index [8], to evaluate the influence of climate on human body. The domestic research of comfort started from 1980s. Dahai Xu and Rong Zhu [9], Jianguo Tan [10], WeiLin Lv [11], Dui Wu [12], Shigong Wang [13] studied the calculating method of human comfort degree from different aspects.

Under the trend of global warming, the probability of extreme weather and climate events greatly increases, and they add more influence on human's survival environment. The living environment and tourism resources draw more and more attention. In this paper, the technologic method and application result of the Human Comfort Degree Forecast System are introduced.

\section{Algorithm}

In this study, the following formula is used in the calculation of human comfort degree [12]:

$$
\mathrm{DI}=1.8 \mathrm{~T}+0.55(1-\mathrm{RH})+32-3.2 \sqrt{\mathrm{V}}
$$

DI (human comfort degree index) is the human comfort degree, $\mathrm{T}$ is average temperature, $\mathrm{RH}$ is relative humidity, and $\mathrm{V}$ is wind speed. In this paper, the human comfort degree is calculated, and then the variation of human comfort degree is analyzed. According to the classification standard of China Meteorological Administration (CMA), human comfort degree is divided into nine grades.

\section{Design and Development of Human Comfort Degree Forecast System}

Technical method. Eq. 1 is used to calculate the human comfort degree. Usually, the low-resolution popular weather forecast products could be used to calculate human comfort degree for the next 24 hours. Here, based on interpreting Numerical Weather Prediction (NWP) products, using inverse distance weighting (IDW) method, the European Centre for Medium-Range Weather Forecasts $\left(\right.$ ECMWF) fine grid NWP product data $\left(0.125^{\circ} \times 0.125^{\circ}\right)$ are interpolated to whole meteorological observatories in Hebei Plain of China. The technical scheme of human comfort degree forecast is shown in Fig. 1. 


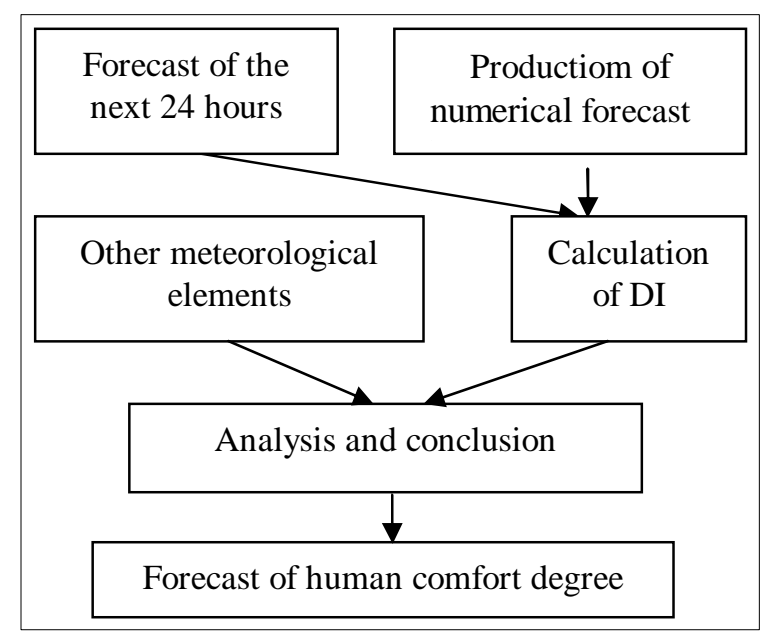

Fig. 1 Technical scheme

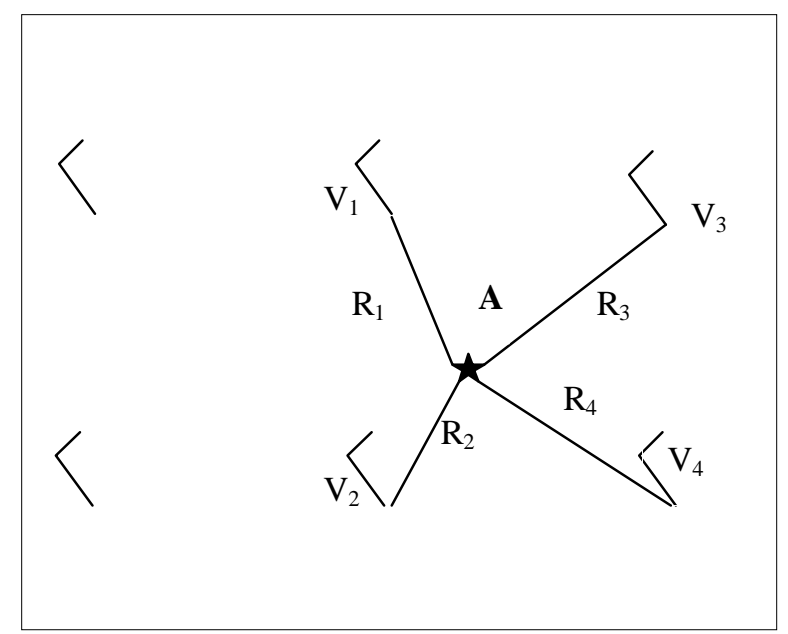

Fig. 2 Diagram of grids and meteorological station

System Development. According to above algorithm and technical method, the human comfort degree forecast system is then developed.

Spatial Interpolation of Meteorological Elements. Spatial interpolation of meteorological elements is based on the projection position of grid data in the plane. A fixed meteorological station is surrounding by four border grid points with known latitude and longitude, and then IDW method can be used to calculate meteorological elements at meteorological station. For example, using interpreting Numerical Weather Prediction (NWP) products of wind speed with 10 meters high, interpolation diagram is shown in Fig. 2. A is the site of meteorological station needed to be interpolated calculation, $V_{1}, V_{2}, V_{3}$ and $V_{4}$ are the nearest grid data of wind speed. $R_{1}, R_{2}, R_{3}$ and $R_{4}$ is the distance from $A$ to $\mathrm{V}_{1}, \mathrm{~V}_{2}, \mathrm{~V}_{3}$ and $\mathrm{V}_{4}$. According to IDW method, wind speed $\mathrm{V}_{\mathrm{A}}$ (10 meters high) can be calculated by Eq. 2.

$$
\mathrm{V}_{\mathrm{A}}=\frac{\frac{\mathrm{V}_{1}}{\mathrm{R}_{1}^{2}}+\frac{\mathrm{V}_{2}}{\mathrm{R}_{2}^{2}}+\frac{\mathrm{V}_{3}}{\mathrm{R}_{3}^{2}}+\frac{\mathrm{V}_{4}}{\mathrm{R}_{4}^{2}}}{\frac{1}{\mathrm{R}_{1}^{2}}+\frac{1}{\mathrm{R}_{2}^{2}}+\frac{1}{\mathrm{R}_{3}^{2}}+\frac{1}{\mathrm{R}_{4}^{2}}}
$$

In this module, temperature, relative humidity and wind speed are calculated in the same way. Other meteorological elements are also calculated, which can provide reference for weather forecast. In this system, the method can be used in other numerical weather forecast products such as T639.

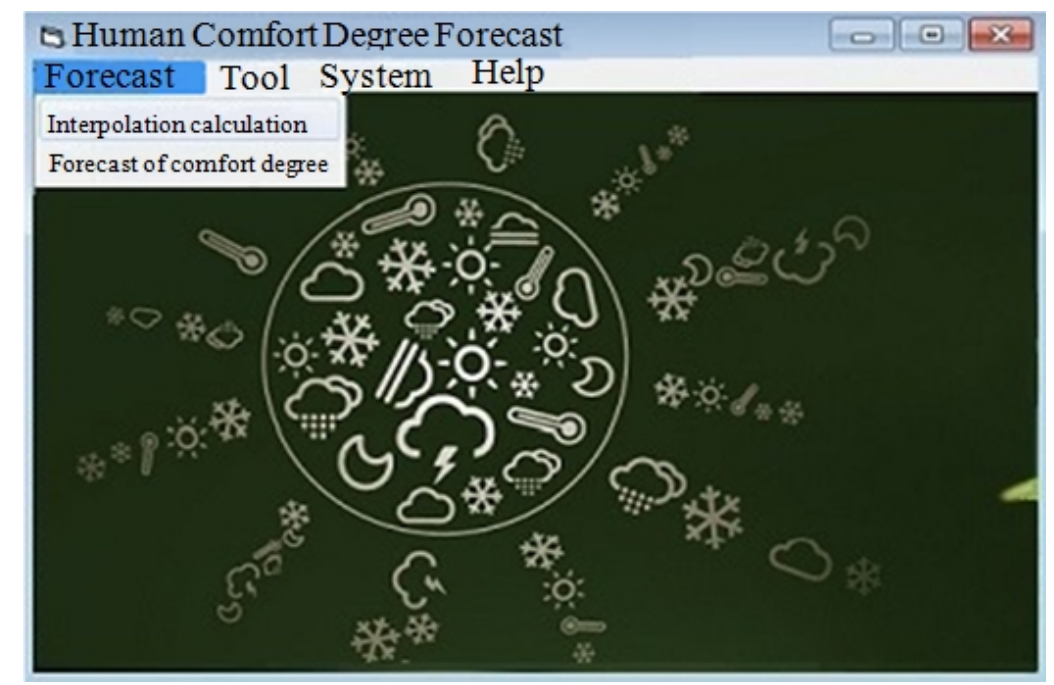

Fig. 3 The main interface of human comfort degree forecast system 
Human Comfort Degree Forecast System Introduction. The system has been developed by Microsoft Visual Basic. The system includes four functional modules which are Forecast, Tool, System and Help (Fig.3). The main Forecast module not only performs calculation and outputs human comfort degree, but other indexes such as wind chill index (in December, January and February) and temperature humidity index (in June, July and August) can be analyzed for human comfort.

\section{Application}

The system is now used for the forecast of human comfort degree in area of Hebei Plain in China, which has the similar geological conditions and climate characteristics.

Fig. 4 gives the case results of human comfort degree forecast for 15 meteorological stations of Shijiazhuang, Zhengding, Zhaoxian, Xingtai, Julu, Linxi, Handan, Feixiang, Daming, Baoding, Mancheng, Rongcheng, Hengshui, Gucheng and Zaoqiang at 1100 BST, 29 July, 2015. The comparison between forecast output and real time calculation of human comfort degree is listed in Table 1.

The preliminary application shows about $73.3 \%$ accuracy between forecast output and real time calculation of human comfort degree.

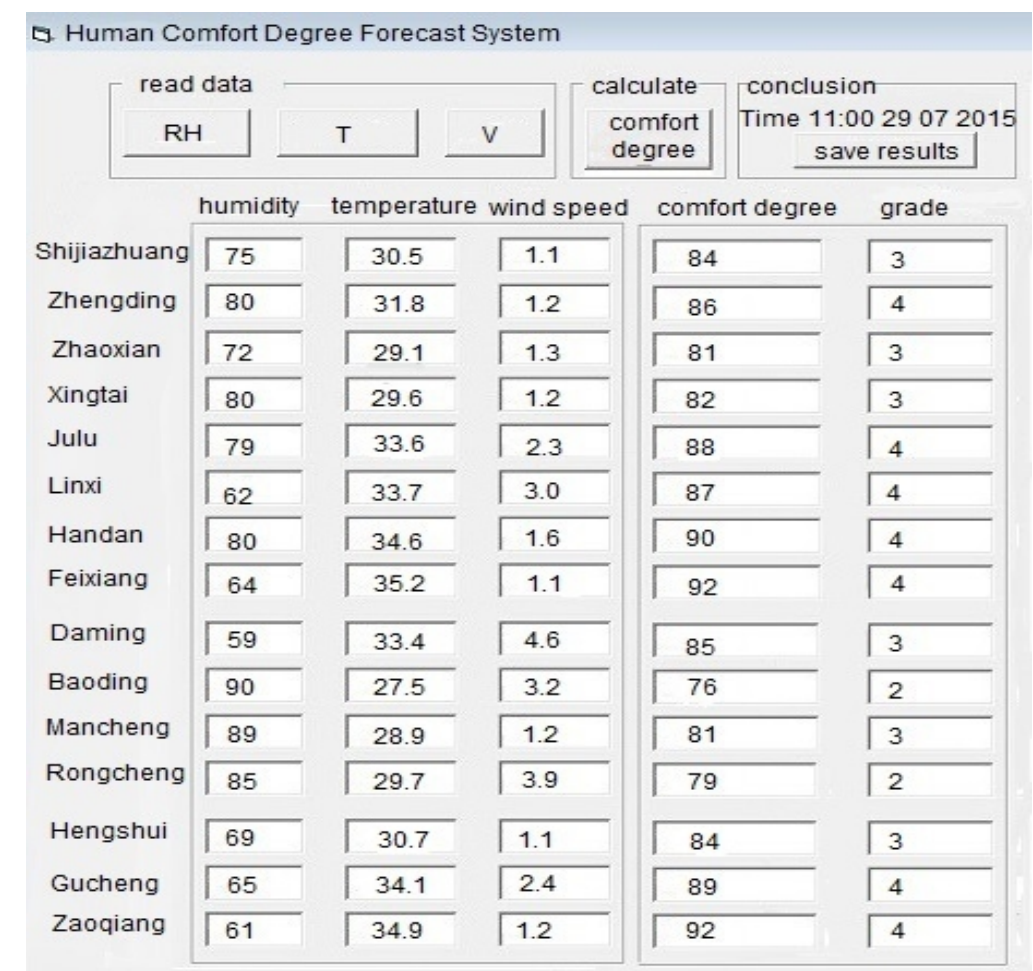

Fig.4 Forecast for 15 meteorological stations at 1100 BST, 29 July, 2015

\section{Summary}

Human comfort degree is a useful index for evaluation of climatic suitability. Based on DI and ECMWF products, the Human Comfort Degree Forecast System has been developed. The preliminary application in Hebei Plain in China shows that there is better agreement between forecast output and real time calculation. The human comfort degree study can provide theoretical foundation and technical support for the development of environmental meteorology, medical meteorology forecast work and for the creation of the livable city. With the development of economic society, air pollution has a serious influence on human health at present. Air quality should be a new factor to human comfort degree. How to consider the impact of air quality is a very important work in the future. 
Table 1 Comparison between forecast output and real time calculation of human comfort degree of 15 meteorological stations at 1100 BST, 29 July, 2015

\begin{tabular}{ccccc}
\hline Meteorological station & Forecast DI & Forecast grade & Real time DI & Real time grade \\
\hline Shijiazhuang & 84 & 3 & 87 & 4 \\
Zhengding & 86 & 4 & 87 & 4 \\
Zhaoxian & 81 & 3 & 85 & 3 \\
Xingtai & 82 & 3 & 87 & 4 \\
Julu & 88 & 4 & 87 & 4 \\
Linxi & 87 & 4 & 86 & 4 \\
Handan & 90 & 4 & 89 & 4 \\
Feixiang & 92 & 4 & 87 & 3 \\
Daming & 85 & 3 & 85 & 2 \\
Baoding & 76 & 3 & 77 & 2 \\
Mancheng & 81 & 2 & 78 & 3 \\
Rongcheng & 79 & 3 & 78 & 3 \\
Hengshui & 84 & 4 & 83 & 4 \\
Gucheng & 89 & 4 & 84 & 86 \\
Zaoqiang & 92 & & & 2 \\
\hline
\end{tabular}

\section{References}

[1] Liu Mei, Yu Bo, The research status, its development and application prospect of human comfort degree, Meteorological Science and Technology. 2002, 30(1):11-18.

[2] Hill L, Griffith OW, Flack M, The measurement of the rate of heat loss at body temperature by convection radiation and evaporation, Philosophical Transactions of Royal Society, 1916, 207:183-220.

[3] Blazejczyk K, Epstein Y, Jendritzky G, et al., Comparison of UTCI to selected thermal indices, International Journal of Biometeorology, 2012, 56:515-535.

[4] Siple A, Passel CF, Measurements of dry atmospheric cooling in subfreezing temperatures, Proceedings of the American Philosophical Society, 1945, 89(1):177-199.

[5] Thom E C, The discomfort index, Weatherwise, 1959, 12:57-60.

[6] Terjung W H, Physiologic Climates of the Conterminous United States: A Bioclimatic Classification Based On man, Annals A.A.G, 1966, 5(1):141-179.

[7] Terjung $\mathrm{W} \mathrm{H}$, World patterns of the distribution of the monthly comfort index, International Journal of Biometeorology, 1968, 12(2):119-151.

[8] Oliver J E, Climate and man's Environment: An Introduction to Applied Climatology, John Wiley and Sons Inc., New York, 1973, pp195-206.

[9] Xu Dahai, Zhu Rong, The sensation of human to temperature \& humility \& wind speed and the clothing, Quarterly Journal of Applied Meteorlolgy, 2000, 11(4):430-439.

[10] Tan Jianguo, Shao Demin, Ma Leiming et al., Human body heat balance model with its application in comfort cndex forecast, Journal of Nanjing Institute of Meteorology, 2001, 24(3):384-390.

[11] Lv Weilin, Research on apparent temperature and calculation method, Beijing Meteorology, 1997(4):23-25.

[12] Wu Dui, Deng Xuejiao, Environmental Meteorology and Special Meteorological Forecasts, China Meteorological Press, Beijing, 2001, pp170-172.

[13] Wang Shigong, Ma Pan, Shang Kezheng, Constructing a calculation method of human comfort degree based on golden section method, China Patent 2012105963660, 2013-05-15. 